\title{
Diclofenac topical gel in excisional wounds maintain heal quality and reduce phlogistic signals ${ }^{1}$
}

\author{
Felipe Lobato da Silva Costa ${ }^{\mathrm{I}}$, Laila Deprá TiussiI ${ }^{\mathrm{II}}$, Mayara Silva Nascimento ${ }^{\mathrm{II}}$, Antonio Carlos de Souza Corrêa ${ }^{\mathrm{II}}$, Edson Yuzur \\ Yasojima ${ }^{\mathrm{III}}$, Carla Andréa Avelar Pires ${ }^{\mathrm{IV}}$
}

DOI: http://dx.doi.org/10.1590/S0102-86502014000500007

${ }^{\mathrm{I}}$ Graduate student, School of Medicine, Para State University (UEPA), Belem-PA, Brazil. Conception and design of the study, manuscript writing, English language.

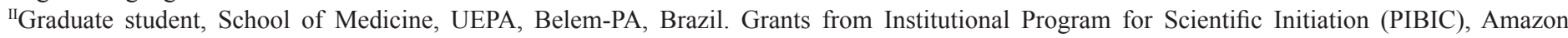
Foundation for Research Funding of Para State (FAPESPA). Acquisition and interpretation of data.

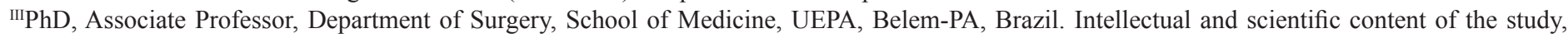
critical revision.

${ }^{\text {IV }} \mathrm{PhD}$, Associate Professor, Department of Dermatology, School of Medicine, UEPA, Belem-PA, Brazil. Conception, design, intellectual and scientific content of the study, critical revision.

\section{ABSTRACT}

PURPOSE: To investigate diclofenac topical gel as an alternative to reduce phlogistic signals and maintain quality of wound repair. METHODS: Fifteen Wistar rats were used in this study; four excisional wounds were performed on the dorsum of each animal. Once in a day, cranial wounds received topical diclofenac gel administration and caudal wounds were washed with isotonic saline. After seven, 14 and 21 postoperative days, five animals were randomly chosen for macroscopic and microscopic wound analysis.

RESULTS: On the $7^{\text {th }}$ day: diclofenac wounds showed significant higher scab formation, however showed less phlogistic signal; diclofenac wounds had larger area and had less neutrophil invasion. On the $14^{\text {th }}$ day: No area difference was noted and diclofenac wounds showed less hyperemia and phlogistic signals; diclofenac wounds showed greater keratinocytes invasion. On the $21^{\text {st }}$ day: Almost all wounds were closed and there were no difference regarding the type of scar formation; diclofenac wounds showed greater monocytes invasion and lower angiogenesis level. No difference was noted in any postoperative day regarding fibroblast invasion, collagen deposit quantity and quality.

CONCLUSION: Diclofenac topical gel is capable of reducing phlogistic signals and do not cause fibroblast or keratinocyte downregulation thus do not lead to excisional wound healing impairment.

Key words: Wound Healing. Regeneration. Cicatrix. Diclofenac. Rats. 


\section{Introduction}

Skin wounds have a unique impact on patient quality of life due to discomfort, bad odor, secretion and fear of pain ${ }^{1}$. When wound repair process is unsatisfactory there is unpleasant scar tissue formation and it might cause harm to body image. In addition, the disease can cause major socioeconomic problems such as absenteeism and even early retirement ${ }^{2}$.

Wounds are loss of skin continuity and can be classified as acute, that usually lead to regular healing; and chronic that often fail to reestablish skin continuity and function ${ }^{3}$. Wound healing is a dynamic and complex cascade, which include several chemical mediators, growth factors, diverse cell types and extracellular matrix substances ${ }^{4}$.

Repair is basically a replacement by connective tissue, which in the permanent state constitutes a scar ${ }^{5}$. It could be dismembered in 5 overlapping phases: coagulation; inflammation; proliferation; wound contraction and remodeling ${ }^{6}$.

Several conditions can interfere in wound healing process: comorbidities such as diabetes, systemic infection; anemia; local infection and necrosis; age; stress; systemic medications. It is notable that healing cascade can be easily deviated from its regular pathway, so it is necessary proper management for open wounds treatment ${ }^{7}$.

Diclofenac is a non-steroidal anti-inflammatory drug (NSAID) that inhibits cyclooxygenases, leading to less formation of prostaglandins that cause inflammation and pain $^{8}$. Diclofenac systemic therapy is commonly used following to hemorrhoidectomy ${ }^{9}$, episiotomy ${ }^{10}$, oral surgery ${ }^{11}$, laparoscopic surgery $^{12}$ for perioperative pain control.

Despite the increasingly use, the effects of systemic diclofenac for perioperative pain are still controversial; several experimental and clinical studies demonstrated that it might be harmful, leading to a poor intestinal anastomosis outcome ${ }^{13}$, delayed tibia fracture healing and can jeopardize other surgical procedures where early tissue healing is crucial ${ }^{14}$.

Experimental studies using rats showed that pre and postoperative systemic diclofenac administration reduces collagen fibers synthesis ${ }^{15}$ and inhibits fibroblasts, leading to a poor wound healing ${ }^{16}$. However, other studies did not report such effect, finding that systemic postoperative diclofenac administration do not interfere on dogs skin incisional wound healing process ${ }^{17}$. Recently, studies using rats also reported that systemic short term use of diclofenac for postoperative pain can cause down regulation of fibroblasts; however, do not interfere significantly on healing process ${ }^{16}$.
NSAID are increasingly used for perioperative pain relief, while their potential effects on wound healing are scarcely investigated ${ }^{17}$. Diclofenac topical administration might be an alternative for avoiding flushing, pain and discomfort in wound area, without having the complications of systemic administration. Moreover topical diclofenac effect in excisional wound healing remains unknown. Thus, diclofenac topical gel was investigated as an alternative to reduce phlogistic signals and maintain quality of wound repair.

\section{Methods}

The project was previously approved by the Animal use and care committee at the Para State University (protocol 01/13). Fifteen (8-10 weeks) male Wistar rats, weighting 250-300g were used in this study. The animals were kept in a vivarium of the Experimental Surgery Laboratory at the Para State University (LCE-UEPA) with a controlled temperature, light, humidity, and noise; water and food was provided ad libitum. The research followed the rules of Brazilian National Law for Animal Care (Law: 11.794/08).

\section{Experimental protocol}

The experimental protocol is illustrated in Figure 1.

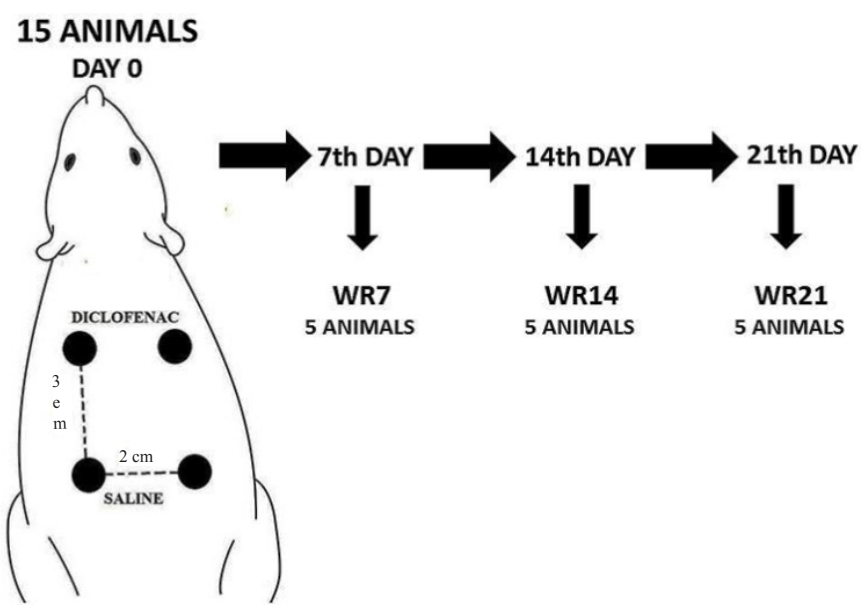

FIGURE 1 - Experimental protocol.

This study is comprised by one group of study, where each animal is its own control:

Wound Repair group (WR): Four spherical wounds were performed on the dorsum of all animals, two each side of median line. In the cranial wounds was done topical diclofenac gel $(11.6 \mathrm{mg} / \mathrm{g}$ ) administration and caudal wounds were only washed with saline isotonic solution $(\mathrm{NaCl} 0.9 \%)$. 
Animals were then randomly assigned into three subgroups $(\mathrm{N}=5$ each): Wound repair seven days (WR7), Wound repair 14 days (WR14) and Wound repair 21 days (WR21) according to the time elapsed from surgery to animal euthanasia.

\section{Surgical procedures}

All surgical procedures were performed under anesthesia (ketamine hydrochloride and xylazine hydrochloride $60 \mathrm{mg} / \mathrm{kg}$ and $6 \mathrm{mg} / \mathrm{kg}$, respectively, i.p.). Dorsal region was shaved using an electric shaver and then antisepsis of region was done.

Excisional wounds were performed using a metallic punch of $4 \mathrm{~mm}$ diameter, reaching skin and subcutaneous tissue. Cranial wounds were $3 \mathrm{~cm}$ distant from caudal wounds. Diclofenac and saline solution were applied everyday and once in a day until euthanasia. Physical restraint was adopted in order to apply the substances. During postoperative days, animals remained in individual cages and isolated from their bedding through a metallic net to avoid wound infection.

Animal euthanasia was achieved by lethal anesthetic doses.

\section{Analyzed parameters}

\section{Gross wound analysis}

Before euthanasia, wounds were analyzed for its visual appearance regarding to scab formation, phlogistic signals and scar formation.

\section{Wound area}

After euthanasia, rats were positioned and pictures of wounds were taken to measure wound area using the software Image $J^{\circledR}$.

\section{Histological analysis}

After euthanasia, wounds were harvested, fixed in $10 \%$ formaldehyde, embedded in paraffin, and stained using Hematoxyline-Eosine (HE) and PicroSirius-red (PS). In HE stained tissues, multiple sections were analyzed with regard to the presence of neutrophils, monocytes, angiogenesis, fibroblasts, collagen deposit and keratinocytes. These assessments were evaluated by a pathologist into: absent (score 0), mild (score 1), moderate (score 2), intense (score 3$)^{18}$. In PS stained tissues, proportion of type I and III collagen fibers was measured.

\section{Statistics}

Analysis of variance (ANOVA), followed by Tukey post- hoc test correction, was performed for the area analysis and proportion of collagen fibers. Kruskal-Wallis test was used for the histological parameters. Statistical significance was assumed at $\mathrm{p}<0.05$.

\section{Results}

Regarding to wound area analysis, our results showed that WR7 had difference between diclofenac wound area and saline solution wound area; other comparisons were not significant (Figure 2).

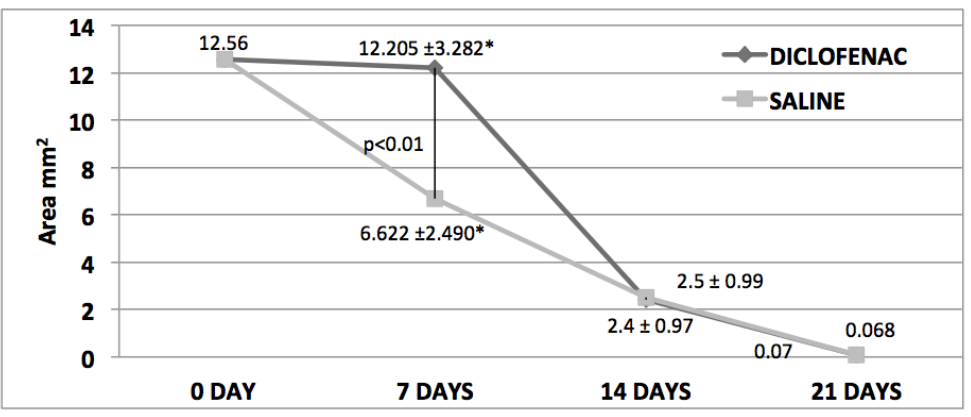

FIGURE 2 - Wound area within days.*ANOVA $\mathrm{p}<0.05$.

Gross wound analysis showed significant higher scab formation in diclofenac wounds at seven days; however showed less phlogistic signal (Figure 3).

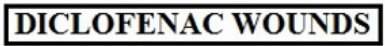

SALINE WOUNDS
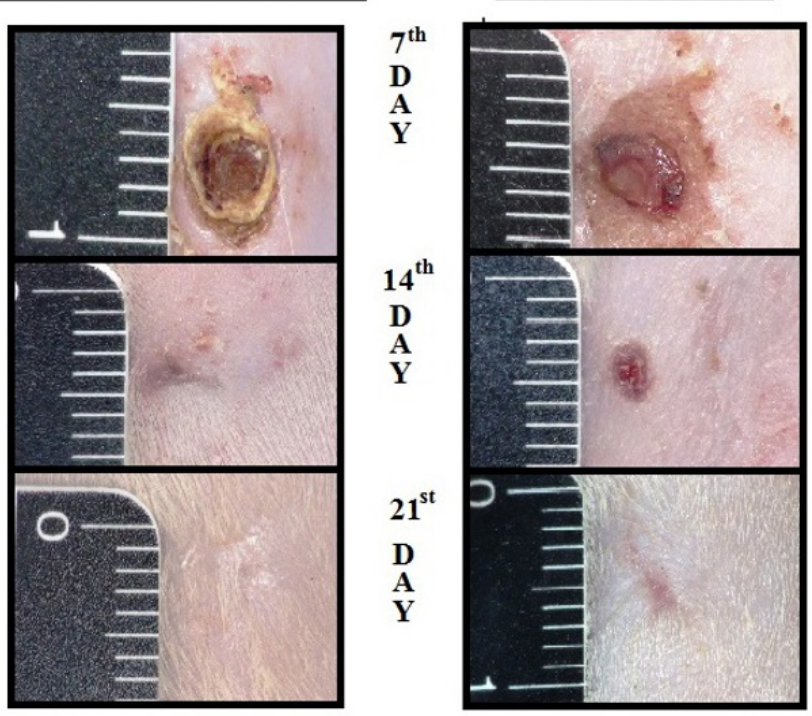

FIGURE 3 - Examples of wounds treated with diclofenac or saline within postoperative days.

At 14 days, wounds showed no difference regarding scab formation; however diclofenac wounds showed less hyperemia and phlogistic signals.

When analyzing the consolidated scar, no difference was noted; diclofenac and saline solution induced to either hypertrophic, hypotrophic and normotrophic scars.

Neutrophils measurement showed difference only between WR7 wounds; other comparisons were not significant (Figure 4). 


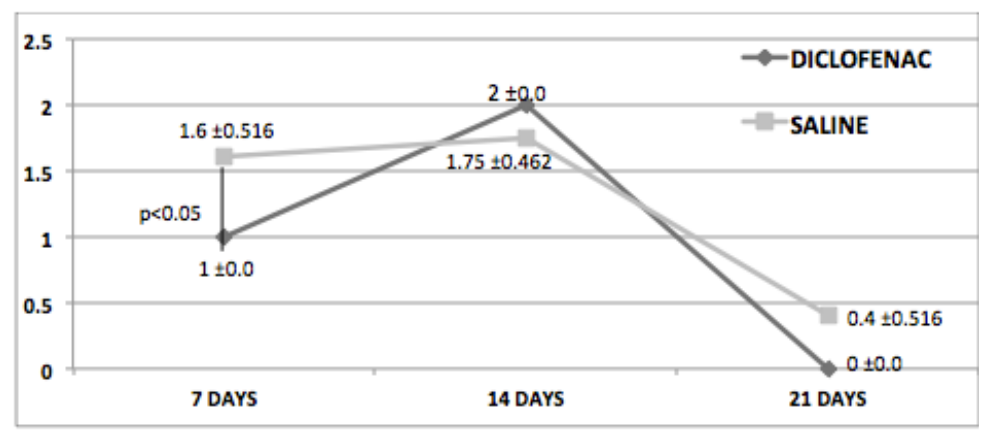

FIGURE 4 - Neutrophils measurement within days. Kruskal-Wallis $\mathrm{p}<0.05$.

Monocytes measurement showed difference only at WR21 wounds; other comparisons were not significant (Figure 5).

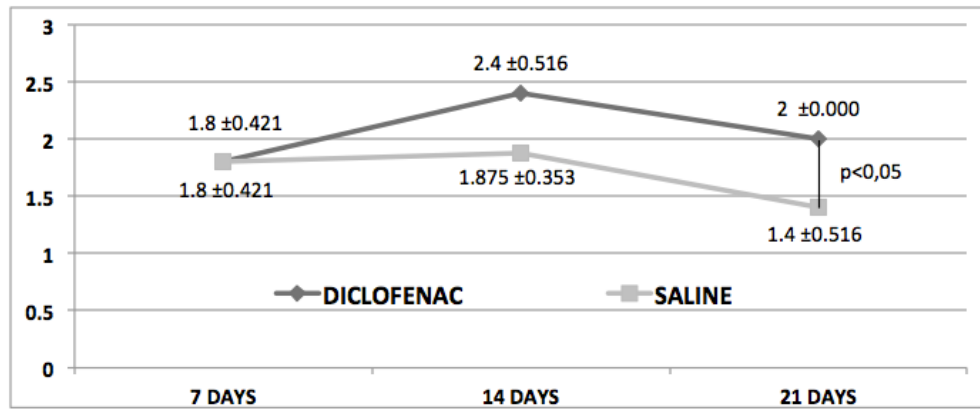

FIGURE 5 - Monocytes measurement within days. Kruskal-Wallis $\mathrm{p}<0.05$.

Angiogenesis was significantly higher on saline WR21 wounds; other comparisons were not significant (Figure 6).

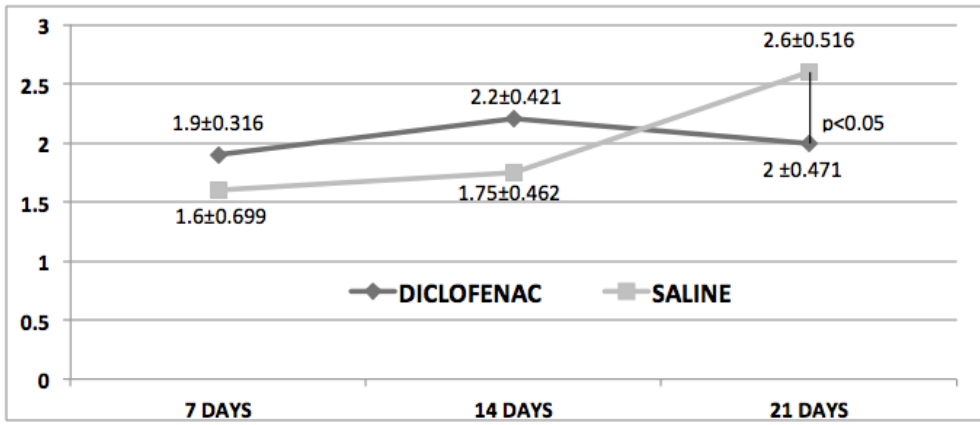

FIGURE 6 - Angiogenesis measurement within days. Kruskal-Wallis $\mathrm{p}<0.05$.

Fibroblast measurement showed similarity between diclofenac and saline treated wounds in all analyzed periods. Collagen fibers deposit showed very similar pattern (Figure 7).

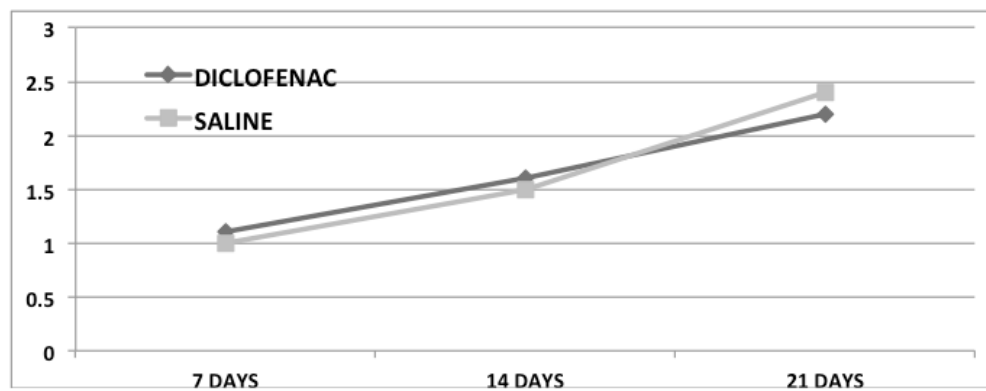

FIGURE 7 - Fibroblast measurement within days. Kruskal-Wallis p $>0.05$.
Regarding keratinocytes migration, WR14 wounds treated by diclofenac showed higher keratinocytes presence in wound area (Figure 8).

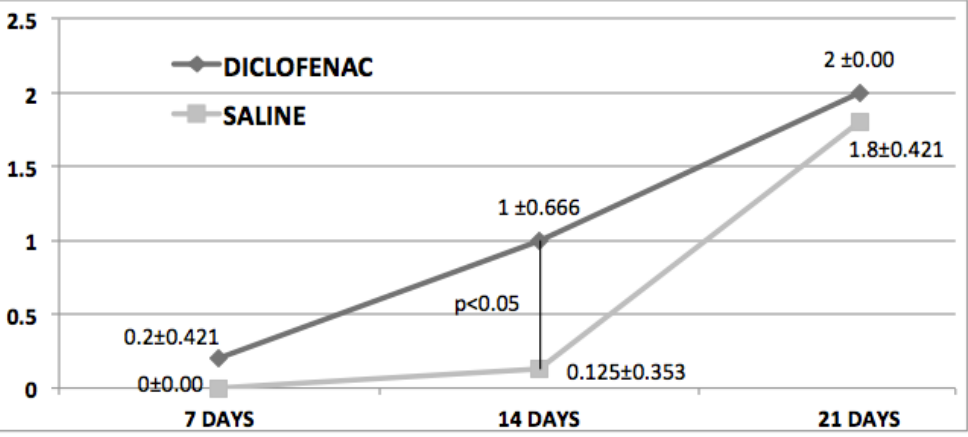

FIGURE 8 - Keratinocytes measurement within days. Kruskal-Wallis $\mathrm{p}<0.05$.

There was no difference regarding the proportion of collagen fibers type I and III in all studied periods. Collagen fibers type I was significantly higher in WR21 wounds when comparing to WR7 wounds (Figure 9).

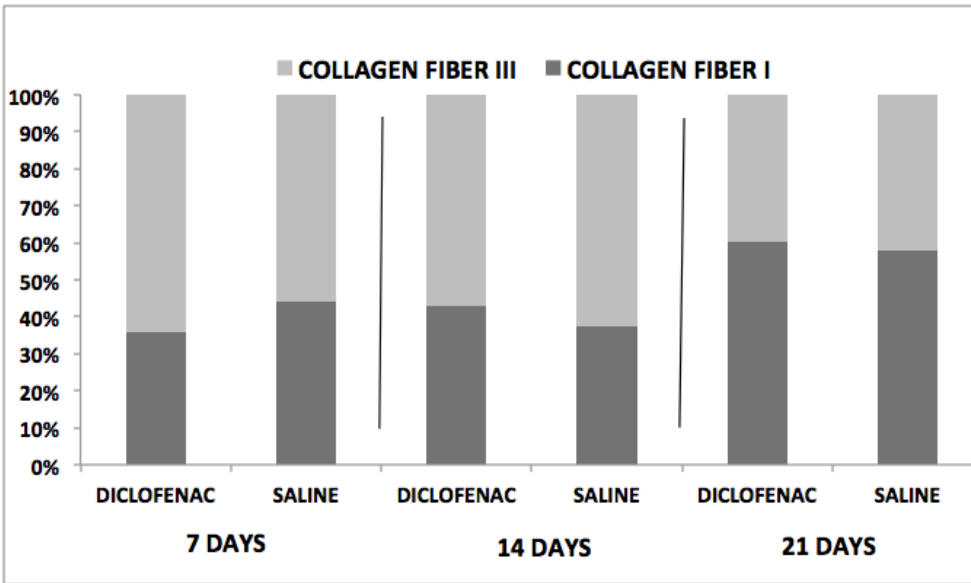

FIGURE 9 - Proportion of collagen fibers type I and III within days. ANOVA $\mathrm{p}>0.05$.

\section{Discussion}

Diclofenac has shown great ability to reduce perioperative pain having morphine-sparing effects, so its use should not be ignored ${ }^{9}$. For skin wounds it is feasible to use diclofenac topical administration, having the potential ability to reduce perioperative pain, since diclofenac has an obvious anti-inflammatory effect as an external preparation, without having systemic complications ${ }^{19}$. Wound healing process can be monitored by assessing the rate of contraction of the wound, period of reepithelialization and histopathology.

The macroscopic analysis showed that diclofenac topical gel causes greater scab formation and reduces phlogistic signals during the first postoperative week. Less phlogistic signals were also seen in diclofenac treated wounds during the second postoperative week and no difference in scar tissue formation 
was seen on the $21^{\text {st }}$ postoperative day. This fact demonstrates the anti-inflammatory ability of diclofenac topical gel in reducing phlogistic symptoms without causing abnormal scar tissue formation; although there is unpleasant greater scab formation during the early phase of wound healing process.

Area analysis showed delayed wound contraction in the first postoperative week when topical diclofenac gel was used. However in the second postoperative week wound area was similar in wounds treated by diclofenac or saline; and on the $21^{\text {st }}$ postoperative day most of wounds were closed, showing that diclofenac did not delay excisional wound heal. From this data we can obviously conclude that diclofenac significantly interfere on wound healing process during the first postoperative week.

Diclofenac anti-proliferative effects in fibroblasts ${ }^{16,20}$ and keratinocytes ${ }^{20}$ were previously described in experimental studies that evaluated systemic diclofenac effect in wound healing. However that effect is unlikely to be an explanation for delayed wound contraction induced by diclofenac in this research, since it happened in an early phase.

The histopathology assessment showed that diclofenac reduced neutrophil migration to wound area only in the first postoperative week. It might be the reason why there was delayed wound contraction in the $7^{\text {th }}$ postoperative day, since neutrophils act removing foreign bodies and devitalized tissue, promoting early debridement. Lack of neutrophils during the early phase of wound healing can cause a prolonged inflammatory step ${ }^{7}$.

Both saline and diclofenac attracted monocytes (macrophages and lymphocytes) to wound area. These cells continue to "clean" the wound and their presence is related to the quantity of foreign bodies in wound area; they also release cytokines that attract fibroblast and endothelial cells. ${ }^{28}$ Was detected that diclofenac treated wounds had more monocytes on the $21^{\text {th }}$ postoperative day, suggesting that diclofenac topical gel administration induce to more foreign bodies presence, which could be explained for the inactive ingredients in diclofenac topical gel formula.

After wound cleaning functions, there is increased angiogenesis, collagen deposit and reepithelialization, leading to the final steps of wound healing. ${ }^{7}$ Angiogenesis is a very important process, since the recently formed blood vessels are responsible for delivering nutrients to wound area and also enables fibroblast and other cell types to reach wound region?

The lower angiogenesis level found in diclofenac treated wounds in the $21^{\text {th }}$ postoperative day, possibly reflect that there was low demand for new blood vessels since the wound healing process was satisfactorily accomplished. On the other hand, saline treated wounds potentially were in need of nutrients income and toxins washout.

During the physiological process of healing, collagen accumulates in the area of the wound until the $21^{\text {st }}$ day after the injury; after this period, the balance between synthesis and degradation of collagen is restored, with a rapid disappearance (apoptosis) of fibroblastic cells. ${ }^{6}$ Fibroblasts level increased within postoperative days; however was not detected any dissimilarity concerning fibroblast presence in wound area when saline or diclofenac was used. Since collagen deposit is directly related to fibroblastic cells action, there was also no difference regarding collagen deposit on wound area.

The fibroblast similar level finding is unique, since previous studies demonstrated that systemic diclofenac can cause fibroblast downregulation, being a serious wound healing impairment factor ${ }^{16,20}$. This fact stands pronounced importance for diclofenac topical gel use in skin wounds as it can potentially reduce phlogistic symptoms and might not cause fibroblast downregulation, leading to a regular wound healing progression.

Synthesis and degradation of collagen is an important process, where collagen fibers type III are constantly being replaced for collagen fibers type I. The latter has more quality and is directly related to wound contraction ${ }^{7}$. When analyzing the proportion of type I/type III collagens fibers, was seen that type I fibers increased within postoperative days; however no difference was observed between diclofenac and saline treated wounds, eliciting that diclofenac topical gel do not impairs quality of collagen deposit.

Reepithelialization is the step straight related to wound closure. Previous studies demonstrated that systemic diclofenac could cause downregulation of keratinocytes, leading to a slower reepithelialization process ${ }^{20}$. The acquired data surprisingly showed that topical diclofenac gel led to keratinocytes up regulation on the $14^{\text {th }}$ postoperative day and did not cause downregulation in the other analyzed periods, leading to early reepithelialization phase. This information also stimulates diclofenac topical gel use as potential drug that could reduce phlogistic signals without causing wound healing impairment.

\section{Conclusion}

Diclofenac topical gel is capable of reducing phlogistic signals and do not cause fibroblast or keratinocyte downregulation thus do not lead to excisional wound healing impairment. 


\section{References}

1. Brown BC, McKenna SP, Solomon M, Wilburn J, McGrouther DA, Bayat A. The patient-reported impact of scars measure: development and validation. Plast Reconstr Surg. 2010;125(5):1439-49.

2. Menck NB, Ward KR, Witten TM, Bochev DG, Diegelman RF. Impaired wound healing. Clin Dermatol. 2007;25(1):19-25.

3. Roldan-Marín R, Contrera-Ruiz J, Arenas R, Vazquez-Del-Mercado E, Caire-Toussanit S, Memize-Veja ME. Fixed sporotrichosis as cause of a chronic ulcera in the knee. Int Wound J. 2009;6(1):63-6.

4. Stroncek JD, Bel N, Reichert WN. Instructional PowerPoint presentation for cutaneous wound healing and tissue response to sutures. J Biomed Mater Res A. 2009;90(4):1230-8.

5. Menetrey J, Kasemkijwattana C, Day CS, Bosch P, Vogt M, Fu FH, Moreland MS, Huad J. Growth Factor Improve Muscle Healing in Vivo. J Bone Joint Surg Br. 2000;82(1):131-7.

6. Clark RAF, Lanigan JM, Dellapelle P, Manseau E, Dvorak HF, Colvin RB. Fibronectin and fibrin provide a provisional matrix for epidermal cell migration during wound reepithelization. J Invest Dermatol. 1982;79(5):264-9.

7. Enoch S, Leaper DJ. Basic science of wound healing. Surgery. 2008;26(2):31-7.

8. Ku EC, Wasvary JM, Cash WD. Diclofenac sodium (GP 45840, Voltaren), a potent inhibitor of prostaglandin synthetase. Biochem Pharmacol. 1975;24(5):641-3.

9. Rahimi M, Kazemeini AR, Pourtabatabaei N, Honarmand AR Comparison of topical anesthetic cream (EMLA) and diclofenac suppository for pain relief after hemorrhoidectomy: a randomized clinical trial. Surg Today. 2012;42(12):1201-5.

10. Altungül AC, Sapmaz E, Kale A. Comparison of diclofenac sodium with indomethacin suppositories for mediolateral episiotomies. Clin Exp Obstet Gynecol. 2012;39(1):112-4.

11. Simone JL, Jorge WA, Horliana AC, Canaval TG, Tortamano IP. Comparative analysis of preemptive analgesic effect of dexamethasone and diclofenac following third molar surgery. Braz Oral Res. 2013;27(3):266-71.

12. Karabayirli S, Demircioğlu RI, Muslu B, Usta B, Sert H, Gözdemir M. The comparative effects of transdermal and intramuscular diclofenac on postlaparoscopic surgery pain. Surg Laparosc Endosc Percutan Tech. 2012;22(4):374-8.

13. van der Vijver RJ, van Laarhoven CJ, Lomme RM, Hendriks T. Diclofenac causes more leakage than naproxen in anastomoses in the small intestine of the rat. Int J Colorectal Dis. 2013;28(9):1209-16.

14. Beck A, Krischak G, Sorg T, Augat P, Farker K, Merkel U, Kinzl L, Claes L. Influence of diclofenac (group of nonsteroidal antiinflammatory drugs) on fracture healing. Arch Orthop Trauma Surg. 2003;123(7):327-32.

15. Tognini JRF, Goldenberg S, Simões MJ, Sauer L, Melo RL, Ortiz PLA. Efeito do diclofenaco de sódio na cicatrização da parede abdominal de ratos. Acta Cir Bras. 1998;13(3):167-71.
16. Krischak GD, Augat P, Claes L, Kinzl L, Beck A. The effects of nonsteroidal anti-inflammatory drug application on incisional wound healing in rats. J Wound Care. 2007;16(2):76-8.

17. Abubakara AA, Maiyea JA, Yakubua AS, Saidub B, Adamuc U, Sahabid SM. Comparative effect of diclofenac sodium and dexamethasone on incisional wound healing in dogs. Sci J Vet Adv. 2012;1(4) 94-100.

18. Garros IC, Campos AC, Tambara EM, Tenorio SB, Torres OJ, Agulham MA. Extract from Passiflora edulis on the healing of open wounds in rats: morphometric and histological study. Acta Cir Bras. 2006;21(3):55-65.

19. Kyuki K, Shibuya T, Tsurumi K, Fujimura H. Anti-inflammatory effect of diclofenac-sodium ointment (cream) in topical application. Jpn J Pharmacol. 1983;33(1):121-32.

20. Harris KL, Bainbridge NJ, Jordan NR, Sharpe JR. The effect of topical analgesics on ex vivo skin growth and human keratinocyte and fibroblast behavior. Wound Repair Regen. 2009;17(3):340-6.

\section{Acknowledgement}

To PhD Robson Domingues, for sharing his laboratory equipment at Morphophysiology Laboratory of Para State University (UEPA).

\section{Correspondence:}

Carla Andréa Avelar Pires

Rua Bernal do Couto, 1802.

66080-200 Belem - PA Brasil

Tel.: (55 91)88262423

choque@1ce.med.br

carlaavelarpires@gmail.com

Received: Dec 17, 2013

Review: Feb 18, 2014

Accepted: March 19, 2014

Conflict of interest: none

Financial source: Amazon Foundation for Research Funding of Para State (FAPESPA).

${ }^{1}$ Research performed at the Experimental Surgery Laboratory, Para State University (LCE-UEPA), Belem-PA, Brazil. 